\title{
Injective objects in triangulated categories
}

Garkusha, Grigory and Prest, Mike 2004

MIMS EPrint: 2006.109

Manchester Institute for Mathematical Sciences

School of Mathematics

The University of Manchester

\footnotetext{
Reports available from: http://eprints.maths.manchester.ac.uk/

And by contacting: The MIMS Secretary

School of Mathematics

The University of Manchester

Manchester, M13 9PL, UK
} 


\title{
INJECTIVE OBJECTS IN TRIANGULATED CATEGORIES
}

\author{
GRIGORY GARKUSHA AND MIKE PREST
}

\section{INTRODUCTION}

We extend ideas and results of Benson and Krause on pure-injectives in triangulated categories. Given a generating set of compact objects in a compactly generated triangulated category $\mathcal{T}$ we define notions of monomorphism, exactness and injectivity relative to this set. We show that the injectives correspond to injective objects in a localisation of the functor category $\operatorname{Mod} \mathcal{T}^{c}$ where $\mathcal{T}^{c}$ denotes the subcategory of compact objects of $\mathcal{T}$. The paper begins by setting up the required localisation theory.

Benson and Krause [BK] showed that injective modules over the Tate cohomology ring of a finite group algebra $k G$, where $k$ is a field of characteristic $p$ and $G$ is a $p$-group, correspond to certain pure-injective objects in the (compactly generated, triangulated) stable module category of $k G$. We generalise this to arbitrary compactly generated triangulated categories, replacing the trivial module $k$ by any compact object and the Tate cohomology ring by the graded endomorphism ring of that object. We obtain the strongest results in the case that this graded endomorphism ring is coherent.

Notation. If no confusion concerning the ring $R$ or the category $\mathcal{C}$ is possible, we usually abbreviate $\operatorname{Hom}_{R}(X, Y)$ or $\operatorname{Hom}_{\mathcal{C}}(X, Y)$ to $(X, Y)$ or $\mathcal{C}(X, Y)$. All subcategories considered are assumed to be full.

\section{Localization, torsion in Grothendieck Categories}

In this section we collect basic facts about localization and torsion in Grothendieck categories. For details and proofs we refer the reader to [Ga] for example.

We say that a subcategory $\mathcal{S}$ of an abelian category $\mathcal{C}$ is a Serre subcategory if for every short exact sequence

$$
0 \longrightarrow X \longrightarrow Y \longrightarrow Z \longrightarrow 0
$$

in $\mathcal{C}$ an object $Y \in \mathcal{S}$ if and only if $X, Z \in \mathcal{S}$. A Serre subcategory $\mathcal{S}$ of a Grothendieck category $\mathcal{C}$ is localizing if it is closed under taking direct limits. Equivalently, the inclusion functor $i: \mathcal{S} \rightarrow \mathcal{C}$ admits a right adjoint $t=t_{\mathcal{S}}: \mathcal{C} \rightarrow \mathcal{S}$ which takes each object $X \in \mathcal{C}$ to the maximal subobject $t(X)$ of $X$ belonging to $\mathcal{S}$. The functor $t$ we call the torsion functor. An object $C$ of $\mathcal{C}$ is said to be $\mathcal{S}$-torsionfree if $t(C)=0$. Given a localizing subcategory $\mathcal{S}$ of $\mathcal{C}$ the quotient category $\mathcal{C} / \mathcal{S}$ consists of those $C \in \mathcal{C}$ such that $(\mathcal{S}, C)=\operatorname{Ext}^{1}(\mathcal{S}, C)=0$.

2000 Mathematics Subject Classification. Primary 18E15; Secondary 18E30.

This paper was written during the visit of the first author to the University of Manchester supported by EPSRC grant GR/R94114/01. He would like to thank the University for the kind hospitality. 
The objects from $\mathcal{C} / \mathcal{S}$ we call $\mathcal{S}$-closed objects. For any $C \in \mathcal{C}$ there exists a canonical exact sequence

$$
0 \longrightarrow A^{\prime} \longrightarrow C \stackrel{\lambda_{C}}{\longrightarrow} C_{\mathcal{S}} \longrightarrow A^{\prime \prime} \longrightarrow 0
$$

with $A^{\prime}=t(C), A^{\prime \prime} \in \mathcal{S}$, and $C_{\mathcal{S}} \in \mathcal{C} / \mathcal{S}$ being the maximal essential extension of $\widetilde{C}=C / t(C)$ such that $C_{\mathcal{S}} / \widetilde{C} \in \mathcal{S}$. The object $C_{\mathcal{S}}$ is unique up to isomorphism and the morphism $\lambda_{C}: C \rightarrow C_{\mathcal{S}}$ is called the $\mathcal{S}$-envelope of $C$. The $\mathcal{S}$-envelope has the property that given any morphism $\alpha: C \rightarrow W$ with $W$ an $\mathcal{S}$-closed object, there is a unique morphism $\alpha_{\S}: C_{\S} \rightarrow W$ such that $\alpha_{\S} \lambda_{C}=\alpha$.

Thus the inclusion functor $i: \mathcal{C} / \mathcal{S} \rightarrow \mathcal{C}$ has the left adjoint localizing functor $(-)_{\mathcal{S}}: \mathcal{C} \rightarrow$ $\mathrm{e} / \mathcal{S}$ which takes each $C \in \mathcal{C}$ to $C_{\mathcal{S}} \in \mathcal{C} / \mathcal{S}$. Then there is an isomorphism

$$
\operatorname{Hom}_{\mathcal{C}}(X, Y) \simeq \operatorname{Hom}_{\mathcal{C} / \mathcal{S}}\left(X_{\mathcal{S}}, Y\right)
$$

for all $X \in \mathcal{C}$ and $Y \in \mathcal{C} / \mathcal{S}$. We note that the functor $(-)_{\mathcal{s}}$ is exact.

\subsection{Finitely presented objects}

An object $X$ of a Grothendieck category $\mathcal{C}$ is finitely generated if whenever there are subobjects $X_{i} \subseteq X$ with $i \in I$ satisfying

$$
X=\sum_{i \in I} X_{i}
$$

then there is a finite subset $J \subset I$ such that

$$
X=\sum_{i \in J} X_{i} .
$$

The subcategory of finitely generated objects is denoted by fg $\mathcal{C}$. A finitely generated object $X$ is said to be finitely presented if every epimorphism $\gamma: Y \rightarrow X$ with $Y \in$ fg $\mathcal{C}$ has finitely generated kernel $\operatorname{Ker} \gamma$. We denote by fp $\mathcal{C}$ the subcategory consisting of finitely presented objects. Finally, we refer to a finitely presented object $X \in \mathcal{C}$ as coherent if every finitely generated subobject of $X$ is finitely presented. The corresponding subcategory of coherent objects will be denoted by coh $\mathcal{C}$.

A Grothendieck category $\mathcal{C}$ is said to be locally finitely generated (respectively, locally finitely presented, locally coherent) if every object $C \in \mathcal{C}$ is a directed sum

$$
C=\sum_{i \in I} C_{i}
$$

of finitely generated subobjects $C_{i}$ (a direct limit of finitely presented, coherent objects, respectively). We note that in a locally coherent category $\mathcal{C}$ the subcategories of finitely presented and coherent objects, fp $\mathcal{C}$ and $\operatorname{coh} \mathcal{C}$, coincide.

If $\mathcal{C}$ is a locally finitely generated category, an object $C$ is finitely generated (finitely presented) iff the representable functor

$$
(C,-): \mathcal{C} \longrightarrow \mathrm{Ab}
$$

respects directed sums (direct limits). 


\subsection{Functor categories}

The main locally finitely presented Grothendieck category we work with is the category $\operatorname{Mod} \mathcal{A}\left(\operatorname{Mod} \mathcal{A}^{\mathrm{op}}\right)$ of contravariant (covariant) functors from a small, or at least skeletally small, preadditive category $\mathcal{A}$ to the category, Ab, of Abelian groups. We also refer to objects of $\operatorname{Mod} \mathcal{A}$ as $\mathcal{A}$-modules. Here we collect some of the basic facts about functor categories: these all follow directly from the definitions.

The Yoneda functor

$$
X \in \mathcal{A} \longmapsto H_{X}=(-, X) \in \operatorname{Mod} \mathcal{A}
$$

takes objects of $\mathcal{A}$ to finitely generated projective objects of $\operatorname{Mod} \mathcal{A}$. Moreover, every finitely generated projective $\mathcal{A}$-module $P$ is a direct summand of a finite coproduct of representable objects $\coprod_{i=1}^{n} H_{X_{i}}$. If $\mathcal{A}$ is an additive category, this direct summand $P$ corresponds to an idempotent of

$$
\operatorname{End}\left(\coprod_{i=1}^{n} H_{X_{i}}\right) \simeq \operatorname{End}\left(\coprod_{i=1}^{n} X_{i}\right) .
$$

If idempotents split in $\mathcal{A}$, this corresponds to a direct summand $X$ of $\coprod_{i=1}^{n} X_{i}$ and then $H_{X} \simeq P$.

We shall denote the subcategory of finitely presented $\mathcal{A}$-modules by $\bmod \mathcal{A}$. Every finitely presented module $M$ has a presentation

$$
\coprod_{i=1}^{n} H_{X_{i}} \longrightarrow \coprod_{j=1}^{m} H_{Y_{j}} \longrightarrow M \longrightarrow 0
$$

by finitely generated projective modules.

If $\mathcal{A}$ is an additive category the category, $\operatorname{Mod} \mathcal{A}$, of $\mathcal{A}$-modules is locally coherent iff $\mathcal{A}$ has pseudo-kernels. A morphism $\varphi: X \rightarrow Y$ is called a pseudo-kernel for $\psi: Y \rightarrow Z$ in $\mathcal{A}$ if the sequence

$$
H_{X} \stackrel{H_{\varphi}}{\longrightarrow} H_{Y} \stackrel{H_{\psi}}{\longrightarrow} H_{Z}
$$

is exact, i.e., every morphism $\delta: Z^{\prime} \rightarrow Y$ with $\psi \delta=0$ factors through $\varphi$. Pseudo-cokernels are defined dually. If $\mathcal{A}$ has kernels, then $\bmod \mathcal{A}$ is an abelian category of projective dimension at most two.

Let $\mathcal{B}$ be an arbitrary subcategory (as always, we mean full) of a preadditive category $\mathcal{A}$ and let $f: \mathcal{B} \rightarrow \mathcal{A}$ denote the inclusion functor. This induces a functor of module categories

$$
f_{*}: \operatorname{Mod} \mathcal{A} \longrightarrow \operatorname{Mod} \mathcal{B},\left.\quad M \longmapsto M\right|_{\mathcal{B}} .
$$

Let

$$
\mathcal{S}=\operatorname{Ker} f_{*}=\{M \in \operatorname{Mod} \mathcal{A} \mid M(\mathcal{B})=0\} .
$$

Then $\mathcal{S}$ is a localizing subcategory and $f_{*}(M)=f_{*}\left(M_{\mathcal{S}}\right)$ for all modules $M$ (see the exact sequence (2.1)). We refer to $\mathcal{S}$ as the annihilator of the subcategory $\mathcal{B}$. If $\mathcal{B}=\{B\}$ consists of a single object, we say that $\mathcal{S}$ is the annihilator of the object $B$.

Theorem $2.1([\mathrm{G}, \mathrm{GG}])$. The functor $f_{*}$ induces an equivalence $\operatorname{Mod} \mathcal{A} / \mathcal{S} \rightarrow \operatorname{Mod} \mathcal{B}$. In particular, if $\mathcal{B}=\{B\}$ consists of a single object $B \in \mathcal{A}$, then $\operatorname{Mod} \mathcal{A} / \mathcal{S}$ is equivalent to the category of $\operatorname{End}_{\mathcal{A}} B$-modules.

We shall, therefore, identify the category of $\mathcal{B}$-modules with the subcategory of $\mathcal{S}$-closed modules in $\operatorname{Mod} \mathcal{A}$. 


\subsection{Derived categories}

In what follows, all functors between triangulated categories are assumed to preserve the triangulated structure. For undefined terms see, for example, $[\mathrm{W}]$.

Let $K^{*}(\mathcal{A})$ and $D^{*}(\mathcal{A}), *=b, \pm, \emptyset$, denote the corresponding homotopy and derived categories of $\operatorname{Mod} \mathcal{A}$. The functor $f_{*}$ induces a functor

$$
\varkappa: K^{*}(\mathcal{A}) \longrightarrow K^{*}(\mathcal{B})
$$

that takes a complex $X=\left(X^{n}, d^{n}\right)$ to $X_{\mathcal{S}}=\left(X_{\mathcal{S}}^{n}, d_{\mathcal{S}}^{n}\right)$ and a morphism $\alpha=\left(\alpha^{n}\right)$ to $\alpha_{\S}=\left(\alpha_{\delta}^{n}\right)$. Since the functor $f_{*}$ is exact there is then an induced functor

$$
\delta: D^{*}(\mathcal{A}) \longrightarrow D^{*}(\mathcal{B})
$$

between the derived categories. Let $\mathcal{T}$ denote $\operatorname{Ker} \delta$ - a thick subcategory of $D^{*}(\mathcal{A})$ (that is, a triangulated subcategory which is closed under direct summands).

Lemma 2.2. The category $\mathcal{T}$ is the subcategory $D_{\mathcal{S}}^{*}(\mathcal{A})$ of $D^{*}(\mathcal{A})$ consisting of all complexes whose cohomology groups belong to $\delta$.

Proof. Any complex $X=\left(X^{n}, d^{n}\right)$ all of whose cohomology groups lie in $\mathcal{S}$ is clearly quasiisomorphic to 0 in $K^{*}(\mathcal{B})$, hence 0 in $D^{*}(\mathcal{B})$. Conversely if $\delta X=0$ then $\varkappa X$ must be quasiisomorphic to 0 in $K^{*}(\mathcal{B})$, so each $H^{n}(\varkappa X)=0$ in $K^{*}(\mathcal{B})$. That is $H^{n}(X)_{\mathcal{S}}=H^{n}\left(X_{\mathcal{S}}\right)=0$. Equivalently, $\left.H^{n}(X)\right|_{\mathcal{B}}=0$.

We have an equivalence

$$
\varphi: D^{*}(\mathcal{A}) / \mathcal{T} \longrightarrow D^{*}(\mathcal{B})
$$

where $D^{*}(\mathcal{A}) / \mathcal{T}$ is the Verdier quotient category of $D^{*}(\mathcal{A})$ with respect to $\mathcal{T}$.

A quasi-inverse to the functor $\varphi$,

$$
\psi: D^{*}(\mathcal{B}) \longrightarrow D^{*}(\mathcal{A}) / \mathcal{T}
$$

is described explicitly as follows.

Let $i: \operatorname{Mod} \mathcal{B}=\operatorname{Mod} \mathcal{A} / \mathcal{S} \rightarrow \operatorname{Mod} \mathcal{A}$ be the inclusion functor. It naturally induces a fully faithful embedding

$$
\iota: K^{*}(\mathcal{B}) \longrightarrow K^{*}(\mathcal{A})
$$

If $\pi: K^{*}(\mathcal{A}) \rightarrow D^{*}(\mathcal{A})$ is the canonical quotient functor, then the values of the composed functor $\pi \iota$ on acyclic complexes in $K^{*}(\mathcal{B})$ belong to $\mathcal{T}$.

So the composed functor

$$
K^{*}(\mathcal{B}) \stackrel{\pi \iota}{\longrightarrow} D^{*}(\mathcal{A}) \longrightarrow D^{*}(\mathcal{A}) / \mathcal{T}
$$

factors as

$$
K^{*}(\mathcal{B}) \stackrel{p}{\longrightarrow} D^{*}(\mathcal{B}) \stackrel{\psi}{\longrightarrow} D^{*}(\mathcal{A}) / \mathcal{T}
$$

where $p: K^{*}(\mathcal{B}) \rightarrow D^{*}(\mathcal{B})$ is the canonical quotient functor. Obviously, $\varphi \psi=1$. On the other hand, let $X$ be a complex of $\mathcal{A}$-modules. There is a morphism $\lambda: X \rightarrow X_{\mathcal{S}}$ of complexes

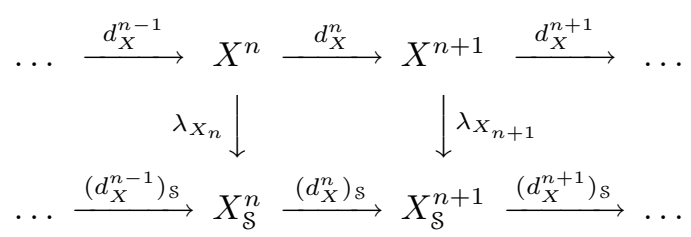


where $\lambda_{X_{n}}$ is the $\mathcal{S}$-envelope of $X_{n}$. The mapping cone of $\lambda$ is the complex

$$
C(\lambda)=X[1] \bigoplus X_{\mathcal{S}}, \quad d_{C(\lambda)}=\left(\begin{array}{cc}
-d_{X} & 0 \\
\lambda & d_{X_{\mathcal{S}}}
\end{array}\right) .
$$

The restriction of $C(\lambda)$ to $\mathcal{B}$ is $C(1)$. The latter complex is null-homotopic. From here it follows that $X$ and $X_{\mathcal{S}}$ are isomorphic in $D(\mathcal{A}) / \mathcal{T}$ and then $\psi \varphi=1$.

Theorem 2.3. Under the conditions of Theorem 2.1, the functor $f_{*}: \operatorname{Mod} \mathcal{A} \rightarrow \operatorname{Mod} \mathcal{B}$ induces an equivalence of categories between $D^{*}(\mathcal{A}) / \mathcal{T}$ and $D^{*}(\mathcal{B}), *=b, \pm, \emptyset$. In particular, if $\mathcal{B}=\{B\}$ consists of a single object $B \in \mathcal{A}$, then the categories $D^{*}(\mathcal{A}) / \mathcal{T}$ and $D^{*}\left(\operatorname{End}_{\mathcal{A}} B\right)$ are naturally equivalent.

If $*=\emptyset$ then the thick subcategory $\mathcal{T}$ of the unbounded derived category $D(\mathcal{A})$ is localizing, that is closed under direct sums. Let $\mathcal{T}^{\perp}$ denote the subcategory of $D(\mathcal{A})$ whose objects $X$ are $\mathcal{T}$-local, i.e. such that $(\mathcal{T}, X)=0$.

Corollary 2.4. The composed functor $D(\mathcal{A}) \rightarrow D(\mathcal{A}) / \mathcal{T} \stackrel{\sim}{\longrightarrow} D(\mathcal{B})$ has a right adjoint. Therefore $D(\mathcal{B})$ can be viewed as the subcategory of $\mathcal{T}$-local objects of $D(\mathcal{A})$.

Proof. By Lemma $2.2 \mathcal{T}$ can be considered as the subcategory $D_{\mathcal{S}}^{*}(\mathcal{A})$ of all complexes whose cohomology groups belong to $\mathcal{S}$. By [F, Theorem 4.1] the inclusion functor $\mathcal{T} \hookrightarrow D(\mathcal{A})$ has a right adjoint. The rest of the proof follows now from [AJS, Proposition 1.6].

\subsection{Inverse limits of categories}

Definition. (1) A limit of a functor $F: I \rightarrow \mathbf{C a t}$, where $I$ is a small category, with values on the "large" category of categories Cat, is a category $\mathcal{C}$ together with functors $\pi_{i}: \mathcal{C} \rightarrow F(i)$ in Cat such that $\pi_{j}=F(\alpha) \pi_{i}$ for any $\alpha: i \rightarrow j$ and which satisfies the universal property: for any category $\mathcal{A} \in$ Cat and any system of compatible functors $f_{i}: \mathcal{A} \rightarrow F(i)$ there exists a unique functor $g: \mathcal{A} \rightarrow \mathcal{C}$ such that $f_{i}=\pi_{i} g$. This universal property guarantees that any two limits of $F$ are equivalent. Below we shall use the notation $\left(\left(\mathfrak{C}_{i}\right), f_{i j}\right)$ to specify values of $F$ on objects and morphisms of $I$ respectively.

(2) Let $I$ be an inversely directed poset and let $\left(\left(\mathcal{C}_{i}\right), f_{i j}\right)$ be the system (of values on objects and morphisms) associated to a functor $F: I \rightarrow$ Cat. The inverse limit of the system is the limit $\left(\mathcal{C}, \pi_{i}\right)_{i \in I}$ of $F$. The category $\mathcal{C}$ will be denoted by $\lim _{i \in I} \mathcal{C}_{i}$. We shall refer to a system indexed by a directed set as an inverse system of categories.

Lemma 2.5. The inverse limit of an inverse system $\left(\left(\mathcal{C}_{i}\right), f_{i j}\right)$ always exists.

Proof. For each $i \in I$ let $p_{i}: \prod \mathcal{C}_{i} \rightarrow \mathcal{C}_{i}$ be the $i$ th projection functor from the productcategory $\prod \mathcal{C}_{i}$. Let $\mathcal{C}$ denote the following subcategory of $\prod \mathcal{C}_{i}$ :

$$
\begin{aligned}
& \text { Ob } \mathcal{C}=\left\{\left(X_{i}\right)_{I} \mid X_{j}=f_{i j}\left(X_{i}\right)\right\} \\
& \text { Mor } \mathcal{C}=\left\{\left(\alpha_{i}\right)_{I} \mid \alpha_{j}=f_{i j}\left(\alpha_{i}\right)\right\}
\end{aligned}
$$

Define $\pi_{i}: \mathcal{C} \rightarrow \mathcal{C}_{i}$ by $\pi_{i}=\left.p_{i}\right|_{\mathcal{e}}$. It is directly verified that $\left(\mathcal{C}, \pi_{i}\right)$ is the inverse limit $\lim _{I} \mathfrak{e}_{i}$.

Let $\operatorname{Mod} \mathcal{A}$ be the module category over a preadditive category $\mathcal{A}$. Let us consider the set $I$ that consists of the localizing subcategories $\mathcal{S}$ of $\operatorname{Mod} \mathcal{A}$ which are annihilators of objects 
$B$ of $\mathcal{A}$. This set is ordered by inclusion. If, moreover, $\mathcal{A}$ is an additive category, then $I$ is a directed set: if $\mathcal{P}$ and $\mathcal{S}$ from $I$ are the annihilators of objects $P$ and $S$ respectively, then the localizing subcategory $\mathcal{T}$ which is the annihilator of $P \coprod S$ is contained both in $\mathcal{P}$ and in $\mathcal{S}$. Let $A_{P}$ and $A_{S}$ denote the endomorphism rings of $P$ and $S$ respectively. If $\mathcal{P} \subseteq \mathcal{S}$ then restriction of the $\mathcal{S}$-localizing functor

$$
(-)_{\mathcal{S}}: \operatorname{Mod} \mathcal{A} \longrightarrow \operatorname{Mod} A_{S}
$$

to $\operatorname{Mod} A_{P}$ induces a functor $f_{\mathcal{P S}}: \operatorname{Mod} A_{P} \rightarrow \operatorname{Mod} A_{S}$. The functor (2.2) factors through $\operatorname{Mod} A_{P}$, because $M_{\mathcal{S}}=\left(M_{\mathcal{P}}\right)_{\mathcal{S}}$ and $\alpha_{\mathcal{S}}=\left(\alpha_{\mathcal{P}}\right)_{\mathcal{S}}$ for any module $M$ and any morphism $\alpha$ of $\operatorname{Mod} \mathcal{A}$. Thus we obtain an inverse system of categories $\left(\left(\operatorname{Mod} \mathcal{A} / \mathcal{P}=\operatorname{Mod} A_{P}\right), f_{\mathcal{P S}}\right)$.

The next result exhibits the generalized module category $\operatorname{Mod} \mathcal{A}$ as an inverse limit of categories of modules over rings and also as an inverse limit of certain localisations.

Proposition 2.6. Let $\mathcal{A}$ be a skeletally small additive category, then $\operatorname{Mod} \mathcal{A}$ is equivalent to the category $\varliminf_{P} \operatorname{Mod} A_{P}$, where $P$ runs over the objects of $\mathcal{A}, A_{P}=\operatorname{End}_{\mathcal{A}} P$, and also is equivalent to the category $\lim _{\mathcal{S} \in I} \operatorname{Mod} A_{S}$ as $\mathcal{S}$ runs over the directed set $I$ constructed above.

Proof. Clearly the two inverse systems of categories are equivalent so we abuse notation by identifying them. The system of localizing functors (2.2) is compatible. Let

$$
\varphi: \operatorname{Mod} \mathcal{A} \longrightarrow \lim _{I} \operatorname{Mod} A_{P}
$$

be a unique functor such that $\pi_{\mathcal{S}} \varphi=(-)_{\mathcal{S}}$ with $\pi_{\mathcal{S}}: \lim _{I} \operatorname{Mod} A_{P} \rightarrow \operatorname{Mod} A_{S}$ the projection corresponding to an element $\mathcal{S}$ of $I$. Clearly, $\operatorname{Ker} \varphi=0$. For an $\mathcal{A}$-module is trivial iff it vanishes on every object of $\mathcal{A}$. Therefore $\varphi$ is a faithful functor.

We now construct a quasi-inverse functor

$$
\psi: \lim _{P} \operatorname{Mod} A_{P} \longrightarrow \operatorname{Mod} \mathcal{A}
$$

to $\varphi$.

Let $\left(X_{P}\right)_{P}$ be an object of $\lim _{P} \operatorname{Mod} A_{P}$. We define the corresponding functor $\psi\left(\left(X_{P}\right)_{P}\right)$ in $\operatorname{Mod} \mathcal{A}$ to have value $X_{P}(P)$ at the object $P$ of $\operatorname{Mod} \mathcal{A}$. On a morphism $\alpha: P \longrightarrow Q$ it takes the value $X_{P \oplus Q}(\alpha)$ where we identify the category $\operatorname{Mod}_{P \oplus Q}$ with the category of functors on the full subcategory of $\mathcal{A}$ with objects $P$ and $Q$.

Given a morphism $\left(\alpha_{P}\right)_{P}:\left(X_{P}\right)_{P} \rightarrow\left(Y_{P}\right)_{P}$ in $\lim _{P} \operatorname{Mod} A_{P}$ we define the value of the corresponding natural transformation $\psi\left(\left(\alpha_{P}\right)_{P}\right)$ at an object $Q$ of $\mathcal{A}$ to be $\alpha_{Q}(Q)$.

One may verify that the above do define functors and morphisms of $\operatorname{Mod} \mathcal{A}$ and that $\psi$ is a quasi-inverse for $\varphi$.

Corollary 2.7. Every object $X$ and every morphism $\alpha$ of $\operatorname{Mod} \mathcal{A}$ with $\mathcal{A}$ an additive category can be presented as

$$
X=\varliminf_{\mathcal{S} \in I} X_{\mathcal{S}} \quad \text { and } \quad \alpha=\varliminf_{\mathcal{S} \in I} \alpha_{\mathcal{S}},
$$

where $I$ is the directed set constructed above, $X_{\mathcal{S}}$ and $\alpha_{\mathcal{S}}$ the corresponding $\mathcal{S}$-localisations of $X$ and $\alpha$.

Example. The category of generalized $R$-modules $\mathcal{C}_{R}=(R \bmod , \mathrm{Ab})$ consists of covariant additive functors defined on the category, $R$ mod, of finitely presented left $R$-modules with values in the category, Ab, of Abelian groups. Given $M \in R \bmod$ we put $S=\operatorname{End}_{R} M$. By 
Theorem 2.1 there exists an equivalence of categories $\mathrm{C}_{R} / \mathcal{S} \stackrel{h}{\rightarrow} \operatorname{Mod} S$ with $\mathcal{S}$ the annihihilator of $M$ and $h\left(F_{\mathcal{S}}\right)=F_{\mathcal{S}}(M)=F(M)$ for all $F \in \mathcal{C}_{R}$. The functor quasi-inverse to $h$ is constructed as follows: $g: \operatorname{Mod} S \rightarrow \mathcal{C}_{R} / \mathcal{S}$ sends an $S$-module $N$ to $\left(\left({ }_{R} M_{S}, N_{S}\right) \otimes_{R}-\right)_{\mathcal{S}}$. In particular, given a functor $F$, we have a natural isomorphism $F_{\mathcal{S}} \simeq\left((M, F(M)) \otimes_{R}-\right)_{\mathcal{S}}$. By the preceding corollary we deduce that

$$
F=\varliminf_{M \in R \bmod }\left((M, F(M)) \otimes_{R}-\right)_{\S} .
$$

\section{Compactly generated triangulated Categories}

We fix a triangulated category $\mathcal{T}$ with arbitrary direct sums. An object $X$ of $\mathcal{T}$ is said to be compact if for every family $\left\{Y_{i}\right\}_{i \in I}$ of objects from $\mathcal{T}$ the canonical map

$$
\bigoplus_{i \in I} \mathcal{T}\left(X, Y_{i}\right) \longrightarrow \mathcal{T}\left(X, \coprod_{i \in I} Y_{i}\right)
$$

is an isomorphism. The suspension of a compact object is compact. The category $\mathcal{T}$ is compactly generated if there exists a small set $\mathcal{C}$ of compact objects of $\mathcal{T}$ such that $\mathcal{T}(\mathcal{C}, Y)=0$ implies $Y=0$, for every object $Y$ in $\mathcal{T}$. We refer to such a set $\mathcal{C}$ as a generating set if it is closed under suspension: for which we write $\mathcal{C}=\Sigma \mathcal{C}$. The triangulated subcategory of $\mathcal{T}$ consisting of compact objects will be denoted by $\mathcal{T}^{c}$. We observe that $\mathcal{T}^{c}$ is the smallest triangulated subcategory in $\mathcal{T}$ containing $\mathcal{C}$ and recall that $\mathcal{T}$ is closed under taking direct products.

The following examples of such categories are important for applications:

(1) the derived category $D(R)$ of unbounded complexes of modules for a ring $R$;

(2) the stable module category $\underline{\operatorname{Mod}} \Lambda$ of a quasi-frobenius $(\mathrm{QF}) \operatorname{ring} \Lambda$;

(3) the stable homotopy category $\mathrm{Ho}(\mathcal{S})$ of $\mathrm{CW}$-spectra.

One can specify in each case generating sets and the compact objects.

(1) The set $\mathcal{R}=\{R[n]\}_{n \in \mathbb{Z}}$ generates $D(R)$, where $R[-n]$ denotes the complex concentrated in the $n$th degree, and the perfect complexes (i.e. the complexes isomorphic to bounded complexes of finitely generated projective modules) are the compact objects in $D(R)$.

(2) If $\left\{S_{1}, \ldots, S_{l}\right\}$ is the set of the simple $\Lambda$-modules, then the set

$$
\mathcal{R}=\left\{\Omega^{n} S_{1}\right\}_{n \in \mathbb{Z}} \bigcup \ldots \bigcup\left\{\Omega^{n} S_{l}\right\}_{n \in \mathbb{Z}}
$$

generates $\underline{\operatorname{Mod}} \Lambda$, and the finitely generated modules are the compact objects in $\operatorname{Mod} \Lambda$.

(3) The set $\mathcal{R}=\left\{S^{n}\right\}_{n \in \mathbb{Z}}$ of suspensions of the sphere spectrum $S^{0}$ generates $\mathrm{Ho}(\mathcal{S})$, and the finite spectra are the compact objects in $\mathrm{Ho}(\mathcal{S})$.

Following Hovey, Palmieri and Strickland [HPS], we call a triangulated category $\mathcal{T}$ monogenic if there is an object $X$ in $\mathcal{T}$ such that the only localizing subcategory in $\mathcal{T}$ containing $X$ is $\mathcal{T}$ itself. We shall refer to $X$ as a generator. All of the above triangulated categories are plainly monogenic.

Definition. Let $\mathcal{R}$ be a set of compact objects in $\mathcal{T}^{c}$ closed under suspension. Consider the functor $H=H_{\mathcal{R}}: \mathcal{T} \rightarrow \operatorname{Mod} \mathcal{R}$ that takes an object $X$ of $\mathcal{T}$ to $H_{X}=\left.\mathcal{T}(-, X)\right|_{\mathcal{R}}$.

(1) A map $X \rightarrow Y$ in $\mathcal{T}$ is said to be an $\mathcal{R}$-monomorphism if the map $H_{X} \rightarrow H_{Y}$ is a monomorphism in $\operatorname{Mod} \mathcal{R}$. 
(2) An object $X$ in $\mathcal{T}$ is called $\mathcal{R}$-injective if every $\mathcal{R}$-monomorphism $\varphi: X \rightarrow Y$ splits, i.e. there exists a map $\psi: Y \rightarrow X$ such that $\psi \varphi=1$.

(3) A triangle $X \rightarrow Y \rightarrow Z \rightarrow \Sigma X$ is $\mathcal{R}$-exact if the sequence $0 \rightarrow H_{X} \rightarrow H_{Y} \rightarrow H_{Z} \rightarrow 0$ is an exact sequence in $\operatorname{Mod} \mathcal{R}$.

The corresponding notions of an $\mathcal{R}$-epimorphism and $\mathcal{R}$-projective object are dually defined. If $\mathcal{R}$ is the subcategory of compact objects $\mathcal{T}^{c}$, the corresponding $\mathcal{R}$-injective, $\mathcal{R}$ projective objects and $\mathcal{R}$-exact triangles will be referred to as pure-injective, pure-projective objects and pure-exact triangles. It follows immediately from the definitions that, for any $\mathcal{R}$, every $\mathcal{R}$-injective ( $\mathcal{R}$-projective) object is pure-injective (pure-projective). Clearly, any product of $\mathcal{R}$-injective objects is $\mathcal{R}$-injective.

Example. (1) Let $\mathcal{R}=\{R[n]\}_{n \in \mathbb{Z}}$ in $D(R)$. Since

$$
H^{n}(X)=D(R)(R[-n], X)
$$

for any complex $X$, we see that $X \rightarrow Y$ is an $\mathcal{R}$-monomorphism in $D(R)$ iff the induced map of cohomology groups $H^{n}(X) \rightarrow H^{n}(Y)$ is a monomorphism for all $n \in \mathbb{Z}$.

It follows that if $X$ is $\mathcal{R}$-injective then each cohomology group $H^{n}(X)$ is an injective $R$-module. To see this note that for each $n$ there is a morphism $X \rightarrow E\left(H^{n}(X)\right)[-n]$, where $E$ denotes injective hull of $R$-modules, with the induced map in $n$-th cohomology being an embedding of $H^{n}(X)$ into its injective hull. Then the map $X \rightarrow \prod_{n \in \mathbb{Z}} E\left(H^{n}(X)\right)[-n]$ is an $\mathcal{R}$-monomorphism which must, therefore, split and hence each embedding $H^{n}(X) \rightarrow$ $E\left(H^{n}(X)\right)$ splits, as required. See the discussion after 3.9 for a complete description of the $\mathcal{R}$-injectives.

(2) A map $M \rightarrow N$ in the stable category $\underline{\operatorname{Mod}} \Lambda$ of a QF-ring $\Lambda$ is an $\mathcal{R}$-monomorphism, where $\mathcal{R}$ is the generating set described earlier, iff the induced map

$$
\widehat{\operatorname{Ext}}_{\Lambda}^{n}(S, M) \longrightarrow \widehat{\operatorname{Ext}}_{\Lambda}^{n}(S, N)
$$

is a monomorphism for all simple modules $S$ and $n \in \mathbb{Z}$.

(3) Let $X$ be a spectrum in $\mathrm{Ho}(\mathcal{S})$ and let $\pi_{n}(X)=\mathrm{Ho}(\mathcal{S})\left(S^{n}, X\right)$ denote its stable homotopy groups. A morphism of spectra $X \rightarrow Y$ is a monomorphism iff the induced map $\pi_{n}(X) \rightarrow \pi_{n}(Y)$ is a monomorphism for all $n \in \mathbb{Z}$.

Now we prove, for general $\mathcal{R}$, results established in [Kr2] for the case $\mathcal{R}=\mathcal{T}^{c}$.

Closely related to the notion of exactness is the concept of phantom map. A map $\varphi$ in $\mathcal{T}$ is said to be $\mathcal{R}$-phantom or, if the context makes $\mathcal{R}$ clear, simply phantom if the map $H_{\varphi}$ in $\operatorname{Mod} \mathcal{R}$ vanishes.

Lemma 3.1. For a triangle $X \stackrel{u}{\longrightarrow} Y \stackrel{v}{\longrightarrow} Z \stackrel{w}{\longrightarrow} \Sigma X$ the following are equivalent:

(1) $u$ is an $\mathcal{R}$-phantom map;

(2) $v$ is an $\mathcal{R}$-monomorphism;

(3) $w$ is an $\mathcal{R}$-epimorphism;

(4) the shifted triangle $Y \stackrel{v}{\longrightarrow} Z \stackrel{w}{\longrightarrow} \Sigma X \stackrel{-\Sigma u}{\longrightarrow} \Sigma Z$ is $\mathcal{R}$-exact.

Proof. Easy.

From Theorem 2.1 it follows that the functor $H: \mathcal{T} \rightarrow \operatorname{Mod} \mathcal{R}$ factors as

$$
\mathcal{T} \stackrel{h}{\longrightarrow} \operatorname{Mod} \mathcal{T}^{c} \stackrel{(-)}{\longrightarrow} \operatorname{Mod} \mathcal{R}
$$


where the functor $h$ takes an object $X$ of $\mathcal{T}$ to the functor $h_{X}=\left.\mathcal{T}(-, X)\right|_{\mathcal{T}^{c}}$ and $(-) \mathcal{s}$ is the localization functor with $\mathcal{S}=\left\{F \in \operatorname{Mod} \mathcal{T}^{c} \mid F(\mathcal{R})=0\right\}$. Moreover, the category $\operatorname{Mod} \mathcal{R}$ is the quotient category $\operatorname{Mod} \mathcal{T}^{c} / \mathcal{S}$. The functor $h$ identifies pure-injectives in $\mathcal{T}$ with injectives in $\operatorname{Mod} \mathcal{T}^{c}$ and the map $\mathcal{T}(X, Y) \rightarrow\left(h_{X}, h_{Y}\right), \vartheta \mapsto h_{\vartheta}$ is an isomorphism iff $Y$ is pure-injective (see [Kr2]).

Below we collect some elementary facts about $\mathcal{R}$-injective objects which we will use later.

Lemma 3.2. The functor $H: \mathcal{T} \rightarrow \operatorname{Mod} \mathcal{R}$ identifies $\mathcal{R}$-injective objects in $\mathcal{T}$ and injective objects in $\operatorname{Mod} \mathcal{R}$.

Proof. Let $M$ be an injective object in $\operatorname{Mod} \mathcal{R}$. Then it is also an injective object in Mod $\mathcal{T}^{c}$. By [Kr2, Lemma 1.7] there exists, up to isomorphism, a unique pure-injective object $X$ in $\mathcal{T}$ such that $M \simeq h_{X}$.

Given an object $Y$ of $\mathcal{T}$, we have

$$
\mathcal{T}(Y, X) \simeq \operatorname{Hom}_{\mathcal{T} c}\left(h_{Y}, h_{X}\right) \simeq \operatorname{Hom}_{\mathcal{R}}\left(\left(h_{Y}\right)_{\mathcal{s}}, H_{X}\right) \simeq \operatorname{Hom}_{\mathcal{R}}\left(H_{Y}, H_{X}\right) .
$$

We use here the fact that $h_{X}$ is injective (for the first isomorphism), that $\left.M\right|_{\mathcal{R}}=\left.h_{X}\right|_{\mathcal{R}}=H_{X}$ and that $M$ is an $\mathcal{S}$-closed object. Clearly, $X$ is $\mathcal{R}$-injective.

Conversely, let $X$ be an $\mathcal{R}$-injective object in $\mathcal{T}$ and $i: H_{X} \rightarrow E$ be a monomorphism with $E$ the injective envelope of $H_{X}$ in $\operatorname{Mod} \mathcal{R}$. We have shown that $E$ is represented by an $\mathcal{R}$-injective object $W$ in $\mathcal{T}$. The isomorphism (3.1) implies that $i=H_{\varphi}$ for some $\varphi: X \rightarrow W$. Since $i$ is a monomorphism, $\varphi$ is an $\mathcal{R}$-monomorphism in $\mathcal{T}$, and so splits. Therefore $i$ splits, and hence $H_{X}$ is an injective object in $\operatorname{Mod} \mathcal{R}$.

Proposition 3.3. The following statements are equivalent for an object $X$ in $\mathcal{T}$ :

(1) $X$ is $\mathcal{R}$-injective;

(2) $H_{X}=\left.\mathcal{T}(-, X)\right|_{\mathcal{R}}$ is an injective object in $\operatorname{Mod} \mathcal{R}$;

(3) the map $\mathcal{T}(Y, X) \rightarrow\left(H_{Y}, H_{X}\right), \vartheta \mapsto H_{\vartheta}$, is an isomorphism for all $Y$ in $\mathcal{T}$;

(4) if $\varphi: Y \rightarrow X$ is an $\mathcal{R}$-phantom map, then $\varphi=0$;

(5) if $\varphi: V \rightarrow W$ is an $\mathcal{R}$-monomorphism, then every map $V \rightarrow X$ factors through $\varphi$.

Proof. The equivalences (1) $\Longleftrightarrow(4) \Longleftrightarrow(5)$ are proved as in [Kr2, Lemma 1.4]. The implication (1) $\Longrightarrow(2)$ follows from the preceding lemma, and (2) $\Longrightarrow$ (3) from (3.1).

(3) $\Longrightarrow$ (4). If $\varphi: Y \rightarrow X$ is an $\mathcal{R}$-phantom map, then $H_{\varphi}=0$.

As regards $\mathcal{R}$-projective objects, one can prove easily that an object $X$ in $\mathcal{T}$ is $\mathcal{R}$-projective iff it is in Add $\mathcal{R}$. The proof of the following lemma is straightforward (see [B, Kr3]).

Lemma 3.4. The functor $H: \mathcal{T} \rightarrow \operatorname{Mod} \mathcal{R}$ identifies $\mathcal{R}$-projective objects in $\mathcal{T}$ and projective objects in $\operatorname{Mod} \mathcal{R}$. Moreover, the map

$$
\mathcal{T}(X, Y) \longrightarrow\left(H_{X}, H_{Y}\right), \quad \vartheta \longmapsto H_{\vartheta},
$$

is an isomorphism for any $\mathcal{R}$-projective object $X$ and any object $Y$ in $\mathcal{T}$.

We consider the localizing subcategory $\mathcal{N}=\langle\mathcal{R}\rangle$ generated by $\mathcal{R}$. This triangulated category is compactly generated because $\mathcal{R}$ consists of compact objects (e.g. see [N, 2.1]). In this case, given an object $X$ in $\mathcal{T}$, there exists a triangle

$$
Y_{X} \longrightarrow X \longrightarrow Z_{X} \longrightarrow \Sigma Y_{X}
$$


with $Y_{X}$ in $\mathcal{N}$ and $Z_{X}$ in $\mathcal{N}^{\perp}=\{W \mid \mathcal{T}(\mathcal{N}, W)=0\}$. Moreover, the inclusion functor $i: \mathcal{N} \rightarrow \mathcal{T}$ has a right adjoint $j: \mathcal{T} \rightarrow \mathcal{N}$ [AJS, Proposition 1.6]. Then an object $X$ of $\mathcal{T}$ is $\mathcal{R}$-injective ( $\mathcal{R}$-projective) iff its image under $j$ is injective (projective) (with respect to $\mathcal{R} \subseteq \mathcal{N}$ ) in $\mathcal{N}$, because $\mathcal{R}$ is a generating set in $\mathcal{N}$.

Note that since the indecomposable injective objects in $\operatorname{Mod} \mathcal{R}$ form a set the indecomposable $\mathcal{R}$-injective objects in $\mathcal{T}$ form a set. We denote this set by $\mathrm{Sp}_{\mathcal{R}} \mathcal{T}$.

The next corollary says that $\mathcal{T}$ has enough $\mathcal{R}$-injectives and enough $\mathcal{R}$-projectives providing $\mathcal{R}$ is a generating set in $\mathcal{T}$. We continue to assume that $\mathcal{R} \subseteq \mathcal{T}^{c}$ and is closed under suspension.

Corollary 3.5. If $\mathcal{R}$ is a generating set in $\mathcal{T}$, then every object $X$ in $\mathcal{T}$ admits a monomorphism $X \rightarrow \prod_{i \in I} E_{i}$ with $E_{i} \in \mathrm{Sp}_{\mathcal{R}} \mathcal{T}$ for all $i$ and an epimorphism $P \rightarrow X$ with $P$ an $\mathcal{R}$-projective object in $\mathcal{T}$. In particular, $\mathcal{T}(X, Y)=0$ for all $Y \in \mathrm{Sp}_{\mathcal{R}} \mathcal{T}$ implies $X=0$.

Proof. The proof of the fact that every object $X$ in $\mathcal{T}$ admits a monomorphism $X \rightarrow$ $\prod_{i \in I} E_{i}$ with $E_{i} \in \mathrm{Sp}_{\mathcal{R}} \mathcal{T}$ is like that of [Kr2, Corollary 1.10]. We take $P$ to be equal to $\bigoplus_{Y \in \mathcal{R}} \bigoplus_{Y \rightarrow X} Y$. Then the induced canonical morphism $P \rightarrow X$ is clearly an epimorphism.

We are interested primarily in the case where $\mathcal{R}$ is a generating set in $\mathcal{T}$. We call a map $\varphi: X \rightarrow Y$ an $\mathcal{R}$-injective envelope of $X$ if $\varphi$ is an $\mathcal{R}$-monomorphism, $Y$ is $\mathcal{R}$-injective and every endomorphism $\psi$ of $Y$ satisfying $\psi \varphi=\varphi$ is an isomorphism.

Lemma 3.6. A map $\varphi: X \rightarrow Y$ is an $\mathcal{R}$-injective envelope of $X$ iff $H_{\varphi}: H_{X} \rightarrow H_{Y}$ is an injective envelope in $\operatorname{Mod} \mathcal{R}$.

Proof. This follows directly from the definitions.

Corollary 3.7. Every object $X$ in $\mathcal{T}$ admits an $\mathcal{R}$-injective envelope $\varphi: X \rightarrow Y$. If $\varphi^{\prime}: X \rightarrow Y^{\prime}$ is another $\mathcal{R}$-injective envelope, then there exists an isomorphism $\psi: Y \rightarrow Y^{\prime}$ such that $\varphi^{\prime}=\psi \varphi$.

Proof. This follows from Proposition 3.3 and the existence of injective envelopes in $\operatorname{Mod} \mathcal{R}$.

The existence of $\mathcal{R}$-injective envelopes in $\mathcal{T}$ implies the existence of a universal $\mathcal{R}$-phantom map $\varphi: X^{\prime} \rightarrow X$ and a universal $\mathcal{R}$-monomorphism $\psi: X \rightarrow X^{\prime \prime}$, as described in the next result, for every object $X$ of $\mathcal{T}$.

Proposition 3.8. Suppose that $\mathcal{R}$ is a generating set for $\mathcal{T}$. Then for every object $X$ in $\mathcal{T}$ there exists, up to isomorphism, a unique triangle

$$
X^{\prime} \stackrel{\varphi}{\longrightarrow} X \stackrel{\psi}{\longrightarrow} X^{\prime \prime} \stackrel{\delta}{\longrightarrow} \Sigma X^{\prime}
$$

having the following properties:

(1) a map $\sigma: Y \rightarrow X$ is an $\mathcal{R}$-phantom map iff $\sigma$ factors through $\varphi$;

(2) every endomorphism $\sigma$ of $X^{\prime}$ satisfying $\varphi=\varphi \sigma$ is an isomorphism.

The same triangle is characterized, up to isomorphism, by the following properties:

(3) a map $\sigma: X \rightarrow Y$ is an $\mathcal{R}$-monomorphism iff $\psi$ factors through $\sigma$;

(4) every endomorphism $\sigma$ of $X^{\prime \prime}$ satisfying $\psi=\sigma \psi$ is an isomorphism. 
Proof. Let $\psi: X \rightarrow X^{\prime \prime}$ be the $\mathcal{R}$-injective envelope of $X$. Complete this map to a triangle

$$
X^{\prime} \stackrel{\varphi}{\longrightarrow} X \stackrel{\psi}{\longrightarrow} X^{\prime \prime} \stackrel{\delta}{\longrightarrow} \Sigma X^{\prime}
$$

The proof of the fact that the triangle satisfies the statements of the proposition is like that of [Kr2, Theorem 1.14].

To illustrate $\mathcal{R}$-injective objects in triangulated categories, consider the derived category $D(R)$ of a ring $R$. The set $\mathcal{R}=\{R[n]\}_{n \in \mathbb{Z}}$ generates $D(R)$. Since

$$
D(R)(R[n], R[m])=\left\{\begin{array}{ccc}
R, & n=m \\
0, & n \neq m
\end{array}\right.
$$

the functor

$$
\begin{aligned}
\operatorname{Mod} \mathcal{R} & (\operatorname{Mod} R)^{\mathbb{Z}}=\prod_{\mathbb{Z}} \operatorname{Mod} R \\
M \longmapsto & \left(M^{n}\right)_{n \in \mathbb{Z}}, \quad M^{n}=M(R[n])
\end{aligned}
$$

is an equivalence of categories.

The functor $H: D(R) \rightarrow \operatorname{Mod} \mathcal{R}=(\operatorname{Mod} R)^{\mathbb{Z}}$ takes a complex $X$ in $D(R)$ to the object $\left(H^{-n}(X)\right)_{n \in \mathbb{Z}}$ in $(\operatorname{Mod} R)^{\mathbb{Z}}$ and we may define the functor $F:(\operatorname{Mod} R)^{\mathbb{Z}} \rightarrow D(R)$ to take an object $\left(M^{n}\right)_{n \in \mathbb{Z}}$ to the complex $M=\left(M^{n}, 0\right)_{n \in \mathbb{Z}}$ with zero differential.

Lemma 3.9. Let $M=\left(M^{n}, 0\right)_{n \in \mathbb{Z}}$ be a complex with zero differential. Then the following relations hold in the derived category $D(R)$ :

$$
M=\coprod_{n \in \mathbb{Z}} M^{n}[-n]=\prod_{n \in \mathbb{Z}} M^{n}[-n],
$$

where $M^{n}[-n]$ is the complex with $M^{n}$ in the $n$th degree and zero in other degrees.

Proof. The natural map

$$
\coprod_{n \in \mathbb{Z}} M^{n}[-n] \longrightarrow \prod_{n \in \mathbb{Z}} M^{n}[-n]
$$

is an $H^{n}$-isomorphism for all $n$. Therefore it is an isomorphism in $D(R)$. Similarly, the natural map

$$
M \longrightarrow \prod_{n \in \mathbb{Z}} M^{n}[-n]
$$

is an $H^{n}$-isomorphism for all $n$.

Obviously, an object $\left(M^{n}\right)_{n \in \mathbb{Z}}$ in $(\operatorname{Mod} R)^{\mathbb{Z}}$ is injective (projective) iff each $M^{n}$ is an injective (projective) $R$-module. Therefore, by 3.3 and 3.4 , a complex $X$ is $\mathcal{R}$-injective $(\mathcal{R}$ projective) in $D(R)$ iff it is a complex of the form $\prod_{\mathbb{Z}} E^{n}[-n]\left(\coprod_{\mathbb{Z}} P^{n}[-n]\right)$ with the $E^{n}$ $\left(P^{n}\right)$ injective (projective) $R$-modules. Thus an $\mathcal{R}$-injective complex is indecomposable iff it is of the form $E[n]$ with $E$ an indecomposable injective module. Therefore,

$$
\mathrm{Sp}_{\mathcal{R}} D(R)=\bigcup_{n \in \mathbb{Z}}(\mathrm{Sp} R)[n],
$$

where $(\mathrm{Sp} R)[n]$ is the set of complexes $E[n]$ concentrated in the $-n$th degree with $E$ an indecomposable injective module.

From Lemma 3.6 it follows that a map $\varphi: X \rightarrow E=\prod_{n \in \mathbb{Z}} E^{n}[-n]$ in $D(R)$ is the $\mathcal{R}$-injective envelope of a complex $X$ iff $H^{n}(\varphi): H^{n}(X) \rightarrow H^{n}(E)=E^{n}$ is the injective envelope of $H^{n}(X)$ in $\operatorname{Mod} R$ for all $n$. Conversely, given a complex $X$ we consider the object 
$\left(H^{n}(X)\right)_{n \in \mathbb{Z}}$ in $(\operatorname{Mod} R)^{\mathbb{Z}}$. Its injective envelope is the morphism $\left(\psi^{n}\right):\left(H^{n}(X)\right)_{\mathbb{Z}} \rightarrow\left(E^{n}\right)_{\mathbb{Z}}$ in $(\operatorname{Mod} R)^{\mathbb{Z}}$ with $\psi^{n}: H^{n}(X) \rightarrow E^{n}$ the injective envelope of $H^{n}(X)$. By Proposition 3.3 and Lemma 3.9 there exists a unique map $\varphi: X \rightarrow \prod_{\mathbb{Z}} E^{n}[-n]$ such that $H^{n}(\varphi)=\psi^{n}$. This $\varphi$ is plainly the $\mathcal{R}$-injective envelope of $X$.

Thus we have obtained an explicit description of the $\mathcal{R}$-injective complexes in $D(R)$ where $\mathcal{R}=\{R[n]\}_{n \in \mathbb{Z}}$. In the next section we extend $[\mathrm{BK}]$ and consider classification of $\mathcal{R}$-injective objects in another type of example.

\section{INJECTIVE OBJECTS}

The class of pure-injective right modules $\operatorname{PInj} R$ of a ring $R$ can be identified via the tensor functor $Q_{R} \mapsto Q \otimes_{R}$ - with injective objects of the category of generalized modules $\mathrm{C}_{R}=(R \bmod , \mathrm{Ab})[\mathrm{GJ}]$. Given any finitely presented module $M$, the category of right $S=\operatorname{End}_{R} M$-modules can be viewed as a full subcategory of the category of $\mathcal{S}$-closed objects in $\mathcal{C}_{R}$, where $\mathcal{S}=\left\{F \in \mathcal{C}_{R} \mid F(M)=0\right\}$, via the functor $N_{S} \mapsto\left(\left({ }_{R} M_{S}, N_{S}\right) \otimes_{R}-\right)_{\mathcal{s}}$ (see the example on p. 6). In particular, this functor induces a map of injective $S$-modules to pure-injective $R$-modules and the latter correspond precisely to the injective objects of the quotient category $\mathfrak{C}_{R} / \mathcal{S}$. So we have the following relation:

$$
\bigcup_{S=\text { End } M} \operatorname{Inj} S \subseteq \text { PInj } R .
$$

The left part of the relation is a proper subclass in PInj $R$ in general. Indeed, by $[\mathrm{H}]$, we have that the image of the indecomposable modules in $\operatorname{Inj} S$, where $S=$ End $M$, in the right Ziegler spectrum of $R$ is the dual of the Ziegler-closed set (in the left Ziegler spectrum of $R$ ) generated by $M$. So if, for instance, $R$ were an artin algebra then the left-hand side would consist exactly of the direct sums of finite-length $R$-modules.

Let $\mathcal{T}$ be a compactly generated triangulated category and let $\mathcal{T}^{c}$ denote the subcategory of compact objects. Roughly speaking, the category of functors Mod $\mathcal{T}^{c}$ plays the same role for the collection, PInj $\mathcal{T}$, of pure-injective objects in $\mathcal{T}$ that the category $\mathcal{C}_{R}$ does for the collection, PInj $R$, of pure-injective modules in $\operatorname{Mod} R$.

In a similar way, one would like to embed injective modules over endomorphism rings of compact objects of $\mathcal{T}$ into PInj $\mathcal{T}$. Precisely, given a compact object $X$ in $\mathcal{T}^{c}$, we consider the $\mathbb{Z}$-graded ring $S=\mathcal{T}(X, X)_{*}=\bigoplus_{n \in \mathbb{Z}} \mathcal{T}\left(\Sigma^{n} X, X\right)$ and the set $\mathcal{R}=\left\{\Sigma^{n} X\right\}_{n \in \mathbb{Z}}$ in $\mathcal{T}^{c}$. By Theorem 2.1 the category, $\operatorname{Mod} \mathcal{R}$, of $\mathcal{R}$-modules is the quotient category of $\operatorname{Mod} \mathcal{T}^{c}$ with respect to the localizing category $\mathcal{S}=\left\{F \in \operatorname{Mod} \mathcal{T}^{c} \mid F(\mathcal{R})=0\right\}$. The category $\operatorname{Mod} \mathcal{T}^{c}$ is equivalent to the inverse limit

$$
\operatorname{Mod} \mathcal{T}^{c} \stackrel{\sim}{\longrightarrow} \lim _{X \in \mathcal{T}^{c}} \operatorname{Mod} \mathcal{R}
$$

of the functor categories $\operatorname{Mod} \mathcal{R}$ where $X$ runs over the objects of $\mathcal{T}^{c}$ and $\mathcal{R}=\left\{\Sigma^{n} X\right\}_{n \in \mathbb{Z}}$ (by the proof of Theorem 2.6). Below we shall generalise results in [BK] and show that the injective $S$-modules may be considered as the injective objects in Mod $\mathcal{R}$. Proposition 3.3 will then give the relation $\operatorname{Inj} S \subseteq$ PInj $\mathcal{T}$, and hence

$$
\bigcup_{S=\mathcal{T}(X, X)_{*}, X \in \mathcal{T}^{c}} \operatorname{lnj} S \subseteq \text { PInj } \mathcal{T} .
$$


We fix a compact object $X$ in $\mathcal{T}^{c}$ and set $S=\mathcal{T}(X, X)_{*}$. Consider the two functors

$$
\Pi: \mathcal{T} \longrightarrow \operatorname{Mod} S, \quad Y \longmapsto \mathcal{T}(X, Y)_{*}=\bigoplus_{n \in \mathbb{Z}} \mathcal{T}\left(\Sigma^{n} X, Y\right)
$$

and

$$
\Upsilon: \operatorname{Mod} S \longrightarrow \operatorname{Func}\left(\mathcal{T}^{\mathrm{op}}, \mathrm{Ab}\right)
$$

that sends any module $M$ to the contravariant functor $\Upsilon_{M}$ given on objects by

$$
\Upsilon_{M}(Y)=\operatorname{Hom}_{S}(\Pi(Y), M)=\operatorname{Hom}_{S}\left(\mathcal{T}(X, Y)_{*}, M\right): \mathcal{T}^{\mathrm{op}} \longrightarrow \mathrm{Ab} .
$$

Clearly the functor $\Pi$ commutes with direct sums. We also observe that $\Pi(\varphi)=0$ where $\varphi: V \rightarrow W$ is a map in $\mathcal{T}$ iff $\varphi$ is an $\mathcal{R}$-phantom map in $\mathcal{T}$.

Below we use the following well-known result (e.g. see [BelK]).

Lemma 4.1. The functor $\Pi: \mathcal{T} \rightarrow \operatorname{Mod} S$ induces an equivalence between the category of $\mathcal{R}$-projective objects (=Add $\mathcal{R}$ ) and the category of projective $S$-modules.

Let $E$ be an injective $S$-module. Then the functor $\Upsilon_{E}$ takes triangles to long exact sequences and it takes direct sums to products. Brown's Representability Theorem in triangulated categories $[\mathrm{N}]$ implies that there exists a representing object $\Gamma_{E}$ and a natural isomorphism

$$
\operatorname{Hom}_{S}\left(\mathcal{T}(X, Y)_{*}, E\right) \simeq \mathcal{T}\left(Y, \Gamma_{E}\right)
$$

for $Y \in \mathcal{T}$. In particular, the graded $S$-module $\mathcal{T}\left(X, \Gamma_{E}\right)_{*}=\Pi\left(\Gamma_{E}\right)$ is isomorphic to $E$. The assignment $E \rightarrow \Gamma_{E}$ yields a fully faithful functor

$$
\Gamma: \operatorname{lnj} S \longrightarrow \mathcal{T} \text {. }
$$

It follows immediately from the isomorphism (4.1) that $\Gamma$ preserves direct products. Let $\ln _{\mathcal{R}} \mathcal{T}$ denote the subcategory in $\mathcal{T}$ consisting of $\mathcal{R}$-injective objects.

Theorem 4.2. The functor $\Gamma$ identifies the class, Inj $S$, of injective $S$-modules $\operatorname{Inj} S$ with the class, $\ln _{\mathcal{R}} \mathcal{T}$, of $\mathcal{R}$-injective objects.

Proof. Given an object $Y \in \mathcal{T}$, the map

$$
\varphi \in \mathcal{T}\left(Y, \Gamma_{E}\right) \longmapsto \Pi(Y) \stackrel{\Pi(\varphi)}{\longrightarrow} \Pi\left(\Gamma_{E}\right) \stackrel{\sim}{\longrightarrow} E \in \operatorname{Hom}_{S}(\Pi(Y), E)
$$

is an isomorphism. Let $\varphi: Y \rightarrow \Gamma_{E}$ be an $\mathcal{R}$-phantom map. Then $\Pi(\varphi)=0$, and hence $\varphi=0$. By Proposition 3.3 the object $\Gamma_{E}$ is $\mathcal{R}$-injective.

Let $E$ be an injective cogenerator in $\operatorname{Mod} S$. It follows from (4.1) that $\mathcal{T}\left(Y, \Gamma_{E}\right) \neq 0$ whenever $\Pi(Y) \neq 0$. Consider the morphism

$$
\varphi: Y \longrightarrow \prod_{Y \rightarrow \Gamma_{E}} \Gamma_{E} .
$$

The homomorphism of $S$-modules

$$
\Pi(\varphi): \Pi(Y) \longrightarrow \prod_{\Pi(Y) \rightarrow E} E
$$

is a monomorphism, and hence $\varphi$ is an $\mathcal{R}$-monomorphism. Here we use the isomorphism (4.1) and the fact that $\Gamma$ preserves direct products. In particular, if $Y \in \operatorname{In}_{\mathcal{R}} \mathcal{T}$ is nonzero then $\Pi(Y) \neq 0$ and $\varphi$ splits. This split map gives an idempotent $e: \prod \Gamma_{E} \rightarrow \prod \Gamma_{E}$. Since 
$\Gamma$ is a fully faithful functor and commutes with products, $e=\Gamma(f)$ for an idempotent $f: \prod E \rightarrow \prod E$. Hence $\operatorname{Im} f$ is $\operatorname{in} \operatorname{Inj} S$, and so $Y=\Gamma_{\operatorname{Im} f} \in \operatorname{Inj}_{\mathcal{R}} \mathcal{T}$.

We discuss a number of consequences. By $\operatorname{lnj} \mathcal{R}$ we also mean the category of injective objects of $\operatorname{Mod} \mathcal{R}$.

Corollary 4.3. The composed functor

$$
H \circ \Gamma: \operatorname{Inj} S \rightarrow \operatorname{lnj} \mathcal{R}, \quad E \longmapsto H_{\Gamma_{E}}=\left.\mathcal{T}\left(-, \Gamma_{E}\right)\right|_{\mathcal{R}}
$$

is an equivalence of categories.

Proof. This follows from Proposition 3.3 and Theorem 4.2.

Let $G$ be a finite group and $k$ be a field of characteristic $p$. The trivial $k G$-module $k$ is a compact object in the stable category $\underline{\operatorname{Mod}} k G$. The Tate cohomology ring $\widehat{H}^{*}(G, k)$ is, by definition, the $\mathbb{Z}$-graded ring $\underline{\operatorname{Hom}}(k, k)_{*}$. In [BK], Benson and Krause classified injective modules over $\widehat{H}^{*}(G, k)$. They showed that $\operatorname{lnj} \widehat{H}^{*}(G, k)$ is equivalent via the functor $\Gamma$ to the category, let us denote it $\mathcal{T}(G, k)$, consisting of the pure-injective $k G$-modules which arise as a direct summand of a (possibly infinite) product of modules of the form $\Omega^{n} k$.

Corollary 4.4. The functors $\Gamma: \operatorname{Inj} \widehat{H}^{*}(G, k) \rightarrow \underline{\operatorname{Mod}} k G$ and $H: \underline{\operatorname{Mod}} k G \rightarrow \operatorname{Mod} \mathcal{R}$, where $\mathcal{R}=\left\{\Omega^{n} k\right\}_{n \in \mathbb{Z}}$, induce an equivalence of categories $\operatorname{Inj} \widehat{H}^{*}(G, k), \mathcal{T}(G, k)$ and $\operatorname{Inj} \mathcal{R}$.

Remark. We note that all $\mathcal{R}$-modules $H_{\Omega^{n} k}$ are injective and together they cogenerate $\operatorname{Mod} \mathcal{R}$, that is $\prod_{n \in \mathbb{Z}} H_{\Omega^{n} k}$ is an injective cogenerator in $\operatorname{Mod} \mathcal{R}$.

We discuss now some properties of the functor $\Upsilon_{M}$.

Corollary 4.5. The following are equivalent for an $S$-module $M$ :

(1) the module $M$ is injective;

(2) the functor $\Upsilon_{M}$ is exact;

(3) the functor $\Upsilon_{M}$ is representable by an object $\Gamma_{M}$ of $\mathcal{T}$, that is $\Upsilon_{M}=\mathcal{T}\left(-, \Gamma_{M}\right)$.

Proof. (1) $\Longrightarrow(2)$ is obvious, $(2) \Longrightarrow(3)$ is a consequence of Brown's Representability Theorem [N], because $\Upsilon_{M}$ takes direct sums to products.

$(3) \Longrightarrow(1)$. Similarly to the proof of Theorem 4.2 one can show that $\Gamma_{M}$ is an $\mathcal{R}$-injective object.

The spectral category Spc $\mathcal{C}$ of an additive category $\mathcal{C}$ has the same objects as $\mathcal{E}$, but the morphisms are given by

$$
\mathcal{e}(X, Y) / \operatorname{rad} \mathcal{C}(X, Y)
$$

where $\operatorname{rad} \mathcal{C}(X, Y)$ consists of the morphisms $\varphi: X \rightarrow Y$ such that for any morphism $\psi: Y \rightarrow X$ the composed morphism $\psi \varphi$ belongs to $\operatorname{rad}(\operatorname{End}(X))$. So

$$
\operatorname{End}_{S_{\mathrm{pc}} \mathcal{C}}(X)=\operatorname{End}_{\mathcal{C}}(X) / \operatorname{rad}\left(\operatorname{End}_{\mathcal{C}}(X)\right) \text {. }
$$

We denote by $\operatorname{Spc} S(\operatorname{Spc} \mathcal{R})$ the spectral category of $\operatorname{Inj} S(\operatorname{Inj} \mathcal{R})$. This spectral category is a Grothendieck category in which every object is injective and projective [GaOb]. In a certain sense, it controls the behavior of the injective objects. We note that two injective $S$-modules are isomorphic iff they are isomorphic in Spc $S$. 
Corollary 4.6. The composed functor $\Gamma \circ H: \operatorname{Spc} S \rightarrow \operatorname{Spc} \mathcal{R}$ is an equivalence of categories.

Proof. This is immediate from Corollary 4.3.

We are now in a position to describe the $\mathcal{R}$-injective objects $\operatorname{lnj}_{\mathcal{R}} \mathcal{T}$ of a monogenic triangulated category $\mathcal{T}$ where $\mathcal{R}=\left\{\Sigma^{n} X\right\}_{n \in \mathbb{Z}}$ where $X$ is a generator of $\mathcal{T}$.

Theorem 4.7. Let $\mathcal{T}$ be a monogenic triangulated category with a generator $X$. Let $\mathcal{R}=$ $\left\{\Sigma^{n} X\right\}_{n}$. Then the functor $\Gamma: \operatorname{Inj} S \rightarrow \mathcal{T}$, where $S$ denotes the graded ring $\mathcal{T}(X, X)_{*}$, identifies injective $S$-modules and $\mathcal{R}$-injective objects of $\mathcal{T}$.

Proof. Combine the preceding statements.

Corollary 4.8. Under the assumptions of Theorem 4.7 the functor $\Gamma: \operatorname{Inj} S \rightarrow \mathcal{T}$ identifies the indecomposable injective $S$-modules $\operatorname{Sp} S$ with the indecomposable $\mathcal{R}$-injective objects in $\mathrm{Sp}_{\mathcal{R}} \mathcal{T}$.

Example. (1) Let $\mathrm{Ho}(\mathcal{S})$ be the homotopy category of CW-spectra. Then the sphere spectrum $S^{0}$ is a generator for $\mathrm{Ho}(\mathcal{S})$. We write $\pi_{*}(S)$ for the graded ring $\mathrm{Ho}(\mathcal{S})\left(S^{0}, S^{0}\right)_{*}$. By the preceding theorem the functor $\Gamma: \operatorname{lnj} \pi_{*}(S) \rightarrow \mathrm{Ho}(\mathcal{S})$ identifies the injective $\pi_{*}(S)$-modules with the $\mathcal{R}$-injective objects of $\mathrm{Ho}(\mathcal{S})$.

(2) Let $\Lambda$ be a quasi-frobenius $k$-algebra, let $\left\{S_{1}, \ldots, S_{n}\right\}$ be the set of distinct simple modules and set $S=\bigoplus_{i=1}^{n} S_{i}$. Then $S$ is a generator for the stable module category $\underline{\operatorname{Mod}} \Lambda$. Denote by $E$ the Tate Ext-algebra $\bigoplus_{n \in \mathbb{Z}} \widehat{\operatorname{Ext}}_{\Lambda}^{n}(S, S)$. Then the functor $\Gamma: \operatorname{lnj} E \rightarrow \underline{\operatorname{Mod}} \Lambda$ identifies the injective $E$-modules with the injective objects of $\underline{\operatorname{Mod}} \Lambda$. In particular, if $\Lambda=k G$ with $G$ a finite $p$-group and $k$ a field of characteristic $p$, then the trivial $k G$-module $k$ is, up to isomorphism, the unique simple $k G$-module and $E$ is the Tate cohomology ring $\widehat{H}^{*}(G, k)$. In this case, the injective objects of $\underline{\operatorname{Mod}} k G$ are the objects of $\mathcal{T}(G, k)$ (see Corollary 4.4).

More generally, given a finitely generated $\Lambda$-module $N$, let $E$ denote the Tate Ext-algebra $\bigoplus_{n \in \mathbb{Z}} \widehat{\operatorname{Ext}}_{\Lambda}^{n}(N, N)$. If $\mathcal{N}$ is the localizing subcategory in $\underline{\operatorname{Mod}} \Lambda$ generated by $N$, then the injective $E$-modules can be identified with the injective objects in $\mathcal{N}$.

In this situation we can also consider the endomorphism ring of a complete resolution $\widehat{P}_{*}$ of $N$. Precisely, let

$$
\widehat{P}_{*}: \quad \ldots \longrightarrow P_{1} \longrightarrow P_{0} \stackrel{\delta}{\longrightarrow} P_{-1} \longrightarrow P_{-2} \longrightarrow \ldots
$$

be a complete resolution for $N$, that is an exact sequence of projective=injective modules such that $\operatorname{Im} \delta \simeq N$.

Denote by $A$ the cochain complex

$$
A=\operatorname{Hom}_{\Lambda}\left(\widehat{P}_{*}, \widehat{P}_{*}\right):
$$

here

$$
A^{n}=\prod_{i} \operatorname{Hom}_{\Lambda}\left(P_{n+i}, P_{i}\right)
$$

with differential $d: A \rightarrow A$ defined by

$$
(d f)(x)=\partial(f(x))-(-1)^{n} f(\partial(x))
$$


where $\partial$ is the differential of $\widehat{P}_{*}$. Then $A$ is a differential graded algebra whose cohomology is the Tate Ext-algebra $E$. By Keller's theorem $[\mathrm{K}]$, there is a triangle equivalence between the localizing subcategory $\mathcal{N}$ and the derived category of differential graded $A$-modules.

As a consequence of Theorem 4.7, the $\mathcal{R}$-injective envelope of an object $Y$ in $\mathcal{T}$ is constructed as follows. Let $\psi: \Pi(Y) \rightarrow E$ be the injective envelope in Mod $S$. By (4.1) there exists a unique map $\varphi: Y \rightarrow \Gamma_{E}$ such that $\Pi(\psi)=\varphi$. This $\varphi$ is plainly the injective envelope for $Y$.

Thus we obtain a complete classification of the $\mathcal{R}$-injective objects of $\mathcal{T}$ whenever we have a classification of the injective $S$-modules. Below we discuss a number of special cases.

A Grothendieck category $\operatorname{Mod} \mathcal{A}$ is locally Noetherian if every direct sum of injective objects is injective. Similarly, we refer to a compactly generated triangulated category $\mathcal{T}$ as locally $\mathcal{R}$-Noetherian if every direct sum of $\mathcal{R}$-injective objects in $\mathcal{T}$ is $\mathcal{R}$-injective.

Theorem 4.9. Let $\mathcal{T}$ be a monogenic triangulated category with a generator $X$, let $S$ be the $\mathbb{Z}$-graded ring $\mathcal{T}(X, X)_{*}$ and let $\mathcal{R}=\left\{\Sigma^{n} X\right\}_{n \in \mathbb{Z}}$. The following statements are equivalent:

(1) $\mathcal{T}$ is locally $\mathcal{R}$-Noetherian;

(2) Mod $\mathcal{R}$ is locally Noetherian;

(3) $S$ is a right Noetherian ring;

(4) every $\mathcal{R}$-injective object $Y$ in $\mathcal{T}$ is isomorphic to a direct sum $Y=\bigoplus_{I} Y_{i}$ of indecomposable objects $Y_{i}$.

Proof. The equivalence (1) $\Longleftrightarrow(2)$ is a consequence of Proposition 3.3. (1), (2) $\Longleftrightarrow(4)$ is well-known.

$(1) \Longrightarrow(3)$. Let $\{E\}_{i \in I}$ be a family of injective right $S$-modules. By assumption, the direct sum $\bigoplus_{I} \Gamma_{E_{i}}$ of the $\mathcal{R}$-injective objects $\Gamma_{E_{i}}$ is $\mathcal{R}$-injective. By Theorem 4.7 there exists an injective module $E$ such that $\Gamma_{E}=\bigoplus_{I} \Gamma_{E_{i}}$. Applying the functor $\Pi$, we obtain

$$
E=\Pi\left(\Gamma_{E}\right)=\Pi\left(\bigoplus_{I} \Gamma_{E_{i}}\right)=\bigoplus_{I} \Pi\left(\Gamma_{E_{i}}\right)=\bigoplus_{I} E_{i} .
$$

Thus any direct sum of injective right $S$-modules is injective. Hence $S$ is right Noetherian.

Before completing the proof we need the following lemma.

Lemma 4.10. If $S$ is a right Noetherian ring, then the graded module $\Pi(Y)$ is finitely generated whenever $Y$ is compact.

Proof. The subcategory of those compact objects $Y$ in $\mathcal{T}^{c}$ such that $\Pi(Y)$ is finitely generated is thick. This follows from the fact that $S$ is right noetherian. Since this thick subcategory contains the generator $X$, it coincides with $\mathcal{T}^{c}$.

(3) $\Longrightarrow(1)$. Let $E=\bigoplus_{I} E_{i}$ with the $E_{i}$ injective $S$-modules. It suffices to show that the canonical map $\bigoplus_{I} \Gamma_{E_{i}} \rightarrow \Gamma_{E}$ is an isomorphism. For that it is sufficient, by Proposition 3.3, to prove that for every compact object $Y$ the induced map

$$
\varphi: \mathcal{T}\left(Y, \bigoplus \Gamma_{E_{i}}\right) \longrightarrow \mathcal{T}\left(Y, \Gamma_{E}\right)
$$

is an isomorphism. We have an isomorphism

$$
\psi: \bigoplus \mathcal{T}\left(Y, \Gamma_{E_{i}}\right) \longrightarrow \mathcal{T}\left(Y, \bigoplus \Gamma_{E_{i}}\right)
$$


since $Y$ is compact. The composed map $\varphi \psi$ is isomorphic to the canonical map

$$
\gamma: \bigoplus \operatorname{Hom}_{S}\left(\Pi(Y), E_{i}\right) \longrightarrow \operatorname{Hom}_{S}(\Pi(Y), E)
$$

by definition of $\Gamma$. By the preceding lemma the module $\Pi(Y)$ is finitely generated. Therefore $\gamma$ is an isomorphism, and hence $\varphi$ is an isomorphism.

Suppose now that the ring $S$ is commutative and Noetherian. A classification of injective $S$-modules is well-known in this case. Precisely, given a homogeneous prime ideal $\mathfrak{p}$, the injective envelope $I_{\mathfrak{p}}=E(S / \mathfrak{p})$ of the quotient $S / \mathfrak{p}$ is indecomposable, and each indecomposable injective module is isomorphic to a shifted copy $I_{\mathfrak{p}}[n]$ for some prime $\mathfrak{p}$ and $n \in \mathbb{Z}$. Since $S$ is Noetherian, every injective $S$-module is a direct sum of modules of the form $I_{\mathfrak{p}}[n]$.

Corollary 4.11. If the graded ring $S$ is commutative Noetherian, then each $\mathcal{R}$-injective object $Y \in \ln _{\mathcal{R}} \mathcal{T}$ is isomorphic to a direct sum $Y=\bigoplus \Gamma_{I_{\mathfrak{p}}[n]}$ of indecomposable injectives $\Gamma_{I_{\mathfrak{p}}[n]} \in \mathrm{Sp}_{\mathcal{R}} \mathcal{T}$.

\section{The Ziegler And Zariski spectra}

Whenever we deal with a locally coherent Grothendieck category $\mathcal{C}$ one can consider two topologies on the set, $\mathrm{Sp} \mathcal{C}$, of (isomorphism types of) indecomposable injective objects of C: the Ziegler and Zariski topologies. Ziegler [Z] introduced a topology on the set Zsp $R$ of indecomposable pure-injective $R$-modules in model-theoretic terms. The definition may be extended to any locally coherent Grothendieck category, see Herzog $[\mathrm{H}]$ and Krause [Kr1]. The Zariski topology on Zsp $R$ has been introduced by Prest in [Pr1] and generalized to additive finitely presented categories in [Pr2]: it is dual to the Ziegler topology.

By definition, the collection of subsets

$$
\mathcal{O}(X)=\{Q \in \operatorname{Sp} \mathcal{C} \mid \mathcal{C}(X, Q) \neq 0\}
$$

where $X \in \operatorname{coh} \mathcal{C}$, forms a basis of open subsets for the Ziegler topology on Sp $\mathcal{C}$. Each such subset is compact, see [H, Kr1] (though if $\mathcal{C}$ is not a module category the whole space might not be compact). Let $\mathcal{S}=\sqrt{ } X$ denote the smallest Serre subcategory in coh $\mathcal{C}$ containing $X \in \operatorname{coh} \mathcal{C}$ and let

$$
\overrightarrow{\mathcal{S}}=\left\{\lim _{\longrightarrow} X_{i} \mid X_{i} \in \mathcal{S}\right\} .
$$

be the localising subcategory that it generates. Then $\mathcal{O}(X)$ coincides with the set

$$
\mathcal{O}(\mathcal{S})=\left\{Q \in \mathrm{Sp} \mathcal{C} \mid t_{\mathcal{S}}(Q) \neq 0\right\}
$$

where $t_{\mathcal{S}}$ is the torsion functor corresponding to the localizing subcategory $\overrightarrow{\mathcal{S}}[\mathrm{H}]$. Moreover, the map

$$
\mathcal{S} \longmapsto \mathcal{O}(\mathcal{S})
$$

gives a 1-1 correspondence between the set of Serre subcategories in coh $\mathcal{C}$ and the algebra of open sets on Sp C [H, Kr1].

Example. The category of generalized $R$-modules $\mathcal{C}_{R}=(R \bmod , \mathrm{Ab})$ is locally coherent. The functor

$$
? \otimes_{R}-: \operatorname{Mod} R \longrightarrow \mathcal{C}_{R}, \quad Q \longmapsto Q \otimes_{R}-,
$$


identifies the pure-injectives in $\operatorname{Mod} R$ with the injectives in $\mathcal{C}_{R}$. One can introduce the Ziegler topology on Zsp $R$ via the functor $? \otimes_{R}-$. Precisely, the collection of subsets

$$
\mathcal{O}(X)=\left\{Q \in \operatorname{Zsp} R \mid\left(X, Q \otimes_{R}-\right) \neq 0\right\},
$$

where $X$ is a coherent (=finitely presented) object in $\mathcal{C}_{R}$, forms a basis of open subsets for the Ziegler topology on Zsp $R$ [Z, H, Kr1].

For example, the set $\mathrm{Sp} R$ of indecomposable injective $R$-modules is closed in Zsp $R$ iff $R$ is right coherent [PRZ, GG]. In this case, $\operatorname{Mod} R$ is locally coherent and the collection of sets (5.1) determines the Ziegler topology on $\operatorname{Sp} R$. Then the inclusion $\operatorname{Sp} R \hookrightarrow Z \operatorname{sp} R$ is a closed map.

Let $\mathcal{T}$ be a compactly generated triangulated category with $\mathcal{T}^{c}$ the subcategory of compact objects and let Zsp $\mathcal{T}$ be the set of indecomposable pure-injective objects in $\mathcal{T}$. The functor $h: \mathcal{T} \rightarrow \operatorname{Mod} \mathcal{T}^{c}, X \mapsto h_{X}=\mathcal{T}(-, X)$, identifies the pure-injectives in $\mathcal{T}$ with the injectives in Mod $\mathcal{T}^{c}$ [Kr2]. Since Mod $\mathcal{T}^{c}$ is a locally coherent category, one can introduce the Ziegler topology on Zsp $\mathcal{T}$ via the functor $h$. Precisely, the collection of subsets

$$
\mathcal{O}(X)=\left\{Q \in \operatorname{Zsp} \mathcal{T} \mid\left(X, h_{Q}\right) \neq 0\right\},
$$

where $X$ is a coherent (=finitely presented) object in $\operatorname{Mod} \mathcal{T}^{c}$, forms a basis of open subsets for the Ziegler topology on Zsp T. We follow [Kr3] in calling this topological space the Ziegler spectrum of $\mathcal{T}$.

Remark. A covariant functor $F: \mathcal{T} \rightarrow \mathrm{Ab}$ is said to be coherent if there exists an exact sequence

$$
\mathcal{T}(X,-) \longrightarrow \mathcal{T}(Y,-) \longrightarrow F \longrightarrow 0
$$

such that $X$ and $Y$ are compact objects. In [Kr3], Krause defined the Ziegler topology as follows. The basic open subsets for the topology are, by definition, the sets

$$
\mathcal{B}(F)=\{Q \in \mathrm{Zsp} \mathcal{T} \mid F(Q) \neq 0\},
$$

where $F$ is a coherent functor. In fact, this topology coincides with the topology defined by the basic open sets $\mathcal{O}(X)$ (see $[\mathrm{Kr} 3$, section 7$]$ for details).

A localizing subcategory $\mathcal{L}$ in $\operatorname{Mod} \mathcal{T}^{c}$ is of finite type if $\mathcal{L}=\overrightarrow{\mathcal{S}}$ with $\mathcal{S}$ the Serre subcategory $\mathcal{L} \cap \operatorname{coh}\left(\operatorname{Mod} \mathcal{T}^{c}\right)$ in $\operatorname{coh}\left(\operatorname{Mod} \mathcal{T}^{c}\right)$. Equivalently, the inclusion functor $\operatorname{Mod} \mathcal{T}^{c} / \mathcal{L} \rightarrow \operatorname{Mod} \mathcal{T}^{c}$ preserves direct limits, see $[\mathrm{Kr} 1]$.

Theorem 5.1. Let $\mathcal{T}$ be a monogenic triangulated category with a generator $X$, let $S$ be the graded ring $\mathcal{T}(X, X)_{*}$ and set $\mathcal{R}=\left\{\Sigma^{n} X\right\}_{n \in \mathbb{Z}}$. Then the following conditions are equivalent:

(1) $S$ is a right coherent ring;

(2) $\operatorname{Mod} \mathcal{R}$ is a locally coherent category;

(3) for every compact object $Y$ in $\mathcal{T}$ the $S$-module $\Pi(Y)=\mathcal{T}(X, Y)_{*}$ is finitely presented;

(4) for every compact object $Y$ in $\mathcal{T}$ the $S$-module $\Pi(Y)=\mathcal{T}(X, Y)_{*}$ is finitely generated;

(5) the localizing subcategory $\mathcal{S}=\left\{M \in \operatorname{Mod} \mathcal{T}^{c} \mid M(\mathcal{R})=0\right\}$ is of finite type;

(6) the set $\mathcal{U}=\left\{\Gamma_{E} \mid E \in \mathrm{Sp} S\right\}$ is closed in Zsp T.

Proof. (1) $\Longleftrightarrow(2)$. The category of right $S$-modules $\operatorname{Mod} S$ (the functor category $\operatorname{Mod} \mathcal{R}$ ) is locally coherent iff the category add $S(\operatorname{add} \mathcal{R})$ of finitely generated projective modules 
(compact projective objects) has pseudo-kernels. By Lemma 4.1 the categories add $S$ and add $\mathcal{R}$ are naturally equivalent.

$(1) \Longrightarrow(3)$. It suffices to observe that the category $\mathcal{S}$ of compact objects $Y$ such that $\Pi(Y)$ is a finitely presented $S$-module is thick. The fact that $\mathcal{S}$ is triangulated follows from properties of modules over coherent rings. Clearly it is thick and contains the object $X$ and, therefore, coincides with $\mathcal{T}^{c}$.

(3) $\Longrightarrow(4)$. Obvious.

(4) $\Longrightarrow(1) . S$ is right coherent iff the kernel of every map $f: P_{0} \rightarrow P_{1}$ between finitely generated projective right $S$-modules is finitely generated. By Lemma $4.1 P_{i}=\Pi\left(Y_{i}\right)$ and $f=\Pi(\varphi)$ for some compact projective objects $Y_{i}$ in $\mathcal{T}, i=0,1$, and some map $\varphi: Y_{0} \rightarrow Y_{1}$. Complete the map $\varphi$ to a triangle

$$
Y_{-1} \longrightarrow Y_{0} \stackrel{\varphi}{\longrightarrow} Y_{1} \longrightarrow \Sigma Y_{-1} .
$$

Then the sequence of $S$-modules

$$
\Pi\left(Y_{-1}\right) \longrightarrow P_{0} \stackrel{f}{\longrightarrow} P_{1}
$$

is exact. Since the object $Y_{-1}$ is compact and $\Pi\left(Y_{-1}\right)$ is finitely generated by assumption, we see that $\operatorname{Ker} f$ is finitely generated.

$(2) \Longrightarrow(5)$. The family $\left\{h_{Y}=\mathcal{T}(-, Y)\right\}_{Y \in \mathcal{T}^{c}}$ is a generating family of coherent objects in $\operatorname{Mod} \mathcal{T}^{c}$. By $\left[\mathrm{G}\right.$, Theorem 5.14] it suffices to show that each $H_{Y}=\left.\mathcal{T}(-, Y)\right|_{\mathcal{R}}$ is a finitely presented object in $\operatorname{Mod} \mathcal{R}$. As in the proof of the implication $(1) \Longrightarrow(3)$ it suffices to observe that the category of compact objects $Y$ such that $H_{Y}$ is finitely presented is thick in $\mathcal{T}^{c}$.

$(5) \Longrightarrow(2)$. This is a consequence of [H, Theorem 2.16].

$(5) \Longleftrightarrow(6)$. By Lemma 3.2 and Corollary $4.8 \mathrm{Sp} \mathcal{R}=\left\{H_{\Gamma_{E}} \mid E \in \mathrm{Sp} S\right\}$. The rest of the proof follows from [Kr1, Corollary 4.3].

For the remainder of the paper the ring $S$ is assumed to be right coherent. By the preceding theorem the functor category $\operatorname{Mod} \mathcal{R}$ is locally coherent. Therefore we can consider the Ziegler topology both on $\mathrm{Sp} S$ and on $\mathrm{Sp} \mathcal{R}$. Moreover, the Ziegler topology on $\mathrm{Sp} \mathcal{R}$ coincides with the topology induced from Zsp $\mathcal{T}[\mathrm{H}, \mathrm{Kr} 1]$. We denote by

$$
\mathcal{O}(M)=\left\{E \in \operatorname{Sp} S \mid \operatorname{Hom}_{S}(M, E) \neq 0\right\}
$$

the basic open set in $\operatorname{Sp} S$ defined by $M \in \bmod S$ and by

$$
\Omega(F)=\left\{H_{\Gamma_{E}} \in \operatorname{Sp} \mathcal{R} \mid \operatorname{Hom}_{\mathcal{R}}\left(F, H_{\Gamma_{E}}\right) \neq 0\right\}
$$

the basic open set in $\operatorname{Sp} \mathcal{R}$ defined by a coherent (=finitely presented) object $F$ in $\operatorname{Mod} \mathcal{R}$.

Given a coherent object $F \in \operatorname{Mod} \mathcal{R}$ determined (as a cokernel) by a map $H_{\varphi}: H_{X} \rightarrow H_{Y}$ with $X$ and $Y$ compact $\mathcal{R}$-projective objects in $\mathcal{T}$, we denote by $M \in \bmod S$ the cokernel of the map $\Pi(\varphi): \Pi(X) \rightarrow \Pi(Y)$. For $E \in \operatorname{Sp} S$, we have an exact sequence

$$
0 \longrightarrow \operatorname{Hom}_{S}(M, E) \longrightarrow \operatorname{Hom}_{S}(\Pi(Y), E) \longrightarrow \operatorname{Hom}_{S}(\Pi(X), E)
$$

By (4.1) and by Proposition 3.3 this sequence is isomorphic to the exact sequence

$$
0 \longrightarrow \operatorname{Hom}_{\mathcal{R}}\left(F, H_{\Gamma_{E}}\right) \longrightarrow \operatorname{Hom}_{\mathcal{R}}\left(H_{Y}, H_{\Gamma_{E}}\right) \longrightarrow \operatorname{Hom}_{\mathcal{R}}\left(H_{X}, H_{\Gamma_{E}}\right) \text {. }
$$

Thus,

$$
\operatorname{Hom}_{\mathcal{R}}\left(F, H_{\Gamma_{E}}\right) \simeq \operatorname{Hom}_{S}(M, E) .
$$


So the inverse image of $\Omega(F)$ under the bijective map $H \circ \Gamma: \operatorname{Sp} S \rightarrow \operatorname{Sp} \mathcal{R}$ is the open set $\mathcal{O}(M)$.

Conversely, if $M \in \bmod S$ then a finite presentation for $M$ gives rise to a map $H_{\varphi}: H_{X} \rightarrow$ $H_{Y}$ between finitely generated projective objects in $\operatorname{Mod} \mathcal{R}$. Let $F$ denote the cokernel of $H_{\varphi}$. As above, it follows that the image of $\mathcal{O}(M)$ is the open set $\Omega(F)$.

Corollary 5.2. The map $H \circ \Gamma: \operatorname{Sp} S \rightarrow \operatorname{Sp} \mathcal{R}$ is a homeomorphism of spaces.

Given a locally coherent category $\mathcal{C}$ we define the dual Ziegler or Zariski topology Zar $\mathcal{C}$ (Zar $S$ and $\operatorname{Zar} \mathcal{R}$ for $\mathcal{C}=\operatorname{Mod} S$ or $\mathcal{C}=\operatorname{Mod} \mathcal{R}$ ) to have the same underlying set as $\mathrm{Sp} \mathcal{C}$ but to have, as a basis of open sets the complements

$$
\mathcal{U}(X)=\mathcal{O}(X)^{c}=\{E \in \mathrm{Sp} \mathcal{C} \mid \mathcal{C}(X, E)=0, X \in \operatorname{coh} \mathcal{C}\}
$$

of the compact Ziegler-open sets [Pr1, Pr2].

If $R$ is a commutative Noetherian ring, then the (Zariski) spectrum $\operatorname{Spec} R$ is homeomorphic via the map $\mathfrak{p} \mapsto I_{\mathfrak{p}}=E(R / \mathfrak{p})$ to the Zariski spectrum Zar $R$.

Similarly, for a compactly generated triangulated category $\mathcal{T}$ we define the Zariski topology on Zsp $\mathcal{T}$ as the Zariski spectrum on $\operatorname{Sp} \mathcal{T}^{c}=\left\{h_{Q}=\left.\mathcal{T}(-, Q)\right|_{\mathcal{T}^{c}} \mid Q \in\right.$ Zsp $\left.\mathcal{T}\right\}$. This toplogical space we denote by Zar $\mathcal{T}$.

Theorem 5.3. The map $H \circ \Gamma: \operatorname{Zar} S \rightarrow \operatorname{Zar} \mathcal{R}$ is a homeomorphism of spaces. Moreover, $\operatorname{Zar} \mathcal{R}$ coincides with the topology induced from $\mathrm{Zar} \mathcal{T}$.

Proof. The first part of the theorem is proved similarly to Corollary 5.2. Let us show that $\operatorname{Zar} \mathcal{R}$ coincides with the topology induced from $\operatorname{Zar} \mathcal{T}$.

Given a coherent object $Y$ in $\operatorname{Mod} \mathcal{T}^{c}$ let

$$
\mathcal{V}(Y)=\left\{E \in \operatorname{Sp}_{\mathcal{R}} \mathcal{T} \mid \operatorname{Hom}_{\mathcal{T}^{c}}\left(Y, h_{E}\right)=0\right\}
$$

denote a basic Zariski-open subset for the induced from Zar $\mathcal{T}$ topology. Since each $h_{E}$, $E \in \mathrm{Sp}_{\mathcal{R}} \mathcal{T}$, is an $\mathcal{S}$-closed object, it follows that

$$
\operatorname{Hom}_{\mathcal{T} c}\left(Y, h_{E}\right) \simeq \operatorname{Hom}_{\mathcal{R}}\left(Y_{\mathcal{S}}, H_{E}\right)
$$

where $Y_{\mathcal{S}}$ is the $\mathcal{S}$-localization of $Y$. By Theorem $5.1 \mathcal{S}$ is of finite type. Therefore $Y_{\mathcal{S}}$ is a coherent object in $\operatorname{Mod} \mathcal{R}[\mathrm{H}, \mathrm{Kr} 1]$. Consequently, $\mathcal{V}(Y)=\mathcal{U}\left(Y_{\mathcal{S}}\right)$.

Conversely, if $X$ is a coherent object in $\operatorname{Mod} \mathcal{R}$, there exists a coherent object $Y$ in $\operatorname{Mod} \mathcal{T}^{c}$ such that $Y_{\mathcal{S}}=X[\mathrm{H}$, Theorem 2.16]. Therefore $\mathcal{U}(X)=\mathcal{V}(Y)$, as claimed.

Suppose that the graded ring $S$ is commutative Noetherian and Spec $S$ is its (Zariski) spectrum consisting of homogeneous prime ideals.

Corollary 5.4. The map $\operatorname{Spec} S \rightarrow \operatorname{Zar} \mathcal{R}$ which sends a prime $\mathfrak{p}$ to $H_{\Gamma_{I_{\mathfrak{p}}}}=\left.\mathcal{T}\left(-, \Gamma_{I_{\mathfrak{p}}}\right)\right|_{\mathcal{R}}$ is a homeomorphism of spaces.

Proof. This follows from Corollary 4.11 and Theorem 5.3.

\section{REFERENCES}

[AJS] Alonso, L., Jeremias, A., Souto, M. J., Localization in categories of complexes and unbounded resolutions, Canad. J. Math. 52(2) (2000), 225-247.

[B] Beligiannis, A., Relative homological algebra and purity in triangulated categories, J. Algebra 227(1) (2000), 268-361. 
[BelK] Beligiannis, A., Krause, H., Realizing maps between modules over Tate cohomology rings, preprint, 2002.

[BK] Benson, D., Krause, H., Pure injectives and the spectrum of the cohomology ring of a finite group, J. reine angew. Math. 542(1) (2002), 23-51.

[F] Franke, J., On the Brown representability theorem for triangulated categories, Topology 40(4) (2001), 667-680

[Ga] Gabriel, P., Des catégories abeliénnes, Bull. Soc. Math. France 90 (1962), 323-448.

[GaOb] Gabriel, P., Oberst, U., Spektralkategorien und reguläre Ringe im von Neumannschen Sinn, Math. Z. 92 (1966), 389-395.

[G] Garkusha, G., Grothendieck categories, Algebra i Analiz 13(2) (2001), 1-68. (Russian). English transl. in St. Petersburg Math. J. 13(2) (2002).

[GG] Garkusha, G., Generalov, A. I., Grothendieck categories as quotient categories of (Rmod, Ab), Fund. Appl. Math. 7(4) (2001), 983-992. (Russian). (also available at www.math.msu.su/ fpm/rus/k01/k014/k01402h.htm)

[GJ] Gruson, L., Jensen, C. U., Dimensions cohomologiques reliées aux foncteurs $\varliminf^{(i)}$, In Paul Dubreil and Marie-Paule Malliavin Algebra Seminar, Lecture Notes in Mathematics, No. 867 (SpringerVerlag, 1981), 234-294.

[H] Herzog, I., The Ziegler spectrum of a locally coherent Grothendieck category, Proc. London Math. Soc. 74(3) (1997), 503-558.

[HPS] Hovey, M., Palmieri, J. H., Strickland, N. P., Axiomatic stable homotopy theory, Mem. Amer. Math. Soc. 128 (1997), No. 610

[JL] Jensen, C. U., Lenzing, H., Model theoretic algebra: with particular emphasis on fields, rings, modules, Algebra, Logic and Appl. 2, New York etc., Gordon and Breach, 1989.

[K] Keller, B., Deriving DG categories, Ann. Sci. École Norm. Sup., IV Sér., 27(1) (1994), 63-102.

[Kr1] Krause, H., The spectrum of a locally coherent category, J. Pure Appl. Algebra 114(3) (1997), $259-271$.

[Kr2] Smashing subcategories and the telescope conjecture - an algebraic approach, Invent. Math. 139(1) (2000), 99-133.

[Kr3] Coherent functors in stable homotopy theory, Fundamenta Math. 173(1) (2002), 33-56.

[N] Neeman, A., The Grothendieck duality theorem via Bousfield's techniques and Brown representability, J. Amer. Math. Soc. 9(1) (1996), 205-236.

[Pr1] Prest, M., Remarks on elementary duality, Ann. Pure Appl. Logic 62(2) (1993), 183-205.

[Pr2] - The Zariski spectrum of the category of finitely presented modules, submitted.

[PRZ] Prest, M., Rothmaler, Ph., Ziegler, M., Absolutely pure and flat modules and "indiscrete" rings, J. Algebra 174(2) (1995), 349-372.

[W] Weibel, C. A., An introduction to homological algebra, Cambridge Studies in Advanced Mathematics 38, Cambridge University Press, Cambridge, 1995.

[Z] Ziegler, M., Model theory of modules, Ann. Pure Appl. Logic 26 (1984), 149-213.

St. Petersburg State University, Department of Mathematics and Mechanics, Universitetsky Pr. 28, 198504, Stary Peterhof, Russia, current address: Mathematics Section, The Abdus Salam International Centre for Theoretical Physics (ICTP), Strada Costiera 11, I-34014 Trieste, Italy

E-mail address: ggarkusha@mailru.com

Department of Mathematics, University of Manchester, Oxford Road, Manchester, M13 9PL, ENGLAND

E-mail address: mprest@maths.man.ac.uk 